\title{
PEPTIDOS TOXICOS Y NO TOXICOS DE CIANOBACTERIAS EN CUERPOS DE AGUA DULCE DE LA V REGION, CHILE
}

\author{
(Toxic and non toxic cyanobacterial peptides in freshwater bodies from \\ the V Region, Chile).
}

Campos, V. ${ }^{\mathbf{1}}$; Muñoz, D. ${ }^{\mathbf{1}}$; Straube, M. ${ }^{\mathbf{1}}$, Lisperguer, S. ${ }^{\mathbf{1}}$,Weckesser, J. ${ }^{2}$.
${ }^{1}$ Laboratorio de Microbiología Ambiental; Facultad de Ciencias Básicas y Matemáticas,
Pontificia Universidad Católica, Valparaíso, Chile.
${ }^{2}$ Institut für Biologie II, Mikrobiologie, Albert-Ludwigs-Universität, Freiburg i. Br. Germany.

Palabras claves: Cianobacteria, toxinas, florecimientos, contaminación de aguas Key words: Cyanobacteria, toxins, blooms, water pollution.

\section{RESUMEN}

En Chile se ha detectado la presencia de algunos géneros de cianobacterias que pueden producir potentes hepatotoxinas y neurotoxinas, las que pueden ser letales para humanos y animales. En el presente trabajo se determinó la presencia de dos géneros de cianobacterias no tóxicos: Chroococcus y Spirulina; y cuatro géneros de cianobacterias productores de toxina, Anabaena, Anabaenopsis, Microcystis y Oscillatoria en tres diferentes cuerpos de agua de la V Región: Lago Peñuelas (Valparaíso), Tranque Recreo (Villa Alemana) y Embalse Los Aromos (Limache). Además se detectó la presencia de hepatotoxinas por MALDI-TOF MS encontrándose microcistina-RR, -LA, -YR y nodularina en Embalse Los Aromos, microcistina-LA en Tranque Recreo y microcistina-RR y LA en Lago Peñuelas. Adicionalmente en algunas de las muestras se detectó la presencia de péptidos no tóxicos, que presentan actividad biológica tales como aeruginosinas, cianopeptolinas $y$ microgininas. Como estos cuerpos de agua dulce son utilizados para abastecimiento público y para la recreación, es importante diseñar planes de tratamiento y monitoreo para detectar y evitar los riesgos a la salud humana y animal provocado por estos microorganismos.

\section{INTRODUCCION}

Las cianobacterias son microorganismos procariotas, fotosintéticos de amplia distribución, que pueden encontrarse en gran variedad de ambientes, ya sea

Recibido el 6 Noviembre 2007

Aceptado el 20 Diciembre 2007
ABSTRACT

In Chile the presence of some genera of cyanobacteria that may cause potent hepatoxins and neurotoxis has been detected, which may become lethal for man and animal. In this paper the occurrence of two non toxic genera of cyanobacteria: Chroococcus and Spirulina was established along with four genera of toxin-producing cyanobacteria, Anabaena, Anabaenopsis, Microcystis and Oscillatoria in three different masses of water from the $V$ Region: Lago Peñuelas (Valparaíso), Tranque Recreo (Villa Alemana) and Embalse Los Aromos (Limache). Likewise the presence of hepatoxins by MALDI-TOF MS was detected which resulted in the occurrence of microcystina-RR,_LA, $-Y R$ and nodularina in Embalse Los Aromos, microcystina -LA in Tranque Recreo and microcystina -RR and LA in Lago Peñuelas. Moreover, the presence of non toxic peptides developing biological activities such as aeruginosinas, cianopeptolins and microgininines was detected. Considering that these freshwater bodies are intended for public supply and recreational purposes, it is of utmost importance to design treatment and supervising plans in order to detect and prevent risks for human and animal health caused by these microorganisms.

acuáticos o terrestres, o como simbiontes de plantas y hongos (Metcalf \& Codd, 2004). En cuerpos de agua dulce, dependiendo de las condiciones de temperatura, luz y concentración de nutrientes (principalmente N y P), estos microorganismos son capaces de proliferar rápidamente formando lo que se conoce como florecimiento o «bloom» (Bartram et al., 1999). La eutrofización de los cuerpos de agua ha llevado en los últimos años a un aumento en la 
aparición de floraciones, las cuales se han reportado en Asia, América, África, Australia, Antártica y Europa, estimándose que la mitad de ellas son tóxicas (Neumann et al., 2000).

Las principales toxinas de las cianobacterias corresponden a neurotoxinas y hepatotoxinas. Las neurotoxinas actúan inhibiendo la transmisión del impulso nervioso; a través del bloqueo de los canales de sodio, uniéndose a los receptores de la acetilcolina, o impidiendo la degradación de la acetilcolina. Son producidas principalmente por los géneros Anabaena, Microcystis, Oscillatoria, Aphanizomenon y Lyngbya (Carmichael, 1994; Roset, 2001).

Las hepatotoxinas son producidas principalmente por los géneros Anabaena, Nostoc, Oscillatoria, Anabaenopsis, Nodularia, Cilindrospermopsis y Microcystis, y son las más frecuentemente encontradas en los cuerpos de agua dulce (Paerl,1996; Campos et al., 1999). Estas cianotoxinas se unen a las proteínas fosfatasas 1 y 2 A de los hepatocitos, lo que provoca un colapso en la estructura del citoesqueleto, produciendo hemorragias internas (Carmichael, 1994).

El caso más grave de intoxicación humana, ocurrió en la ciudad de Caruaru, Brasil, el año 1994, donde 50 pacientes murieron después de un tratamiento de hemodiálisis de rutina con agua contaminada con toxinas de los géneros Microcystis y Cilindrospermopsis (Carmichel et al., 2001; Jochimsen et al., 2004).

Además de la toxicidad aguda de las hepatotoxinas, diversos estudios han demostrado que el consumo de pequeñas dosis de microcistina-LR puede promover el desarrollo de carcinoma al hígado (Ueno et al., 1996).

En Chile se ha detectado la presencia de cianobacterias que son capaces de producir toxinas en las regiones II, V, VI, VIII, IX, X y en la región Metropolitana, además se ha podido detectar la presencia de las hepatotoxinas: microcistina-RR, -LR, -FR e -YR en la VIII Región, y la presencia de microcistina-RR en la V Región (Campos et al., 2005; Neumann et al., 2000; Campos et al., 1999, Vera, 2005).

En el presente estudio se determinó la presencia de cianobacterias y hepatotoxinas presentes en tres cuerpos de agua dulce de la V Región, Chile: Lago Peñuelas, ubicado en la Ciudad de Valparaíso y el Embalse los Aromos, ubicado en la ciudad de Limache que son empleados tanto como fuente de agua de abastecimiento como recreacional; y el Tranque Recreo, ubicado en la ciudad de Villa Alemana, que es utilizado sólo para uso recreacional.

Las muestras colectadas en cada cuerpo de agua desde Marzo del 2005 a Enero del 2006, fueron analizados por espectrometría de masas (MALDI-TOF), para determinar la presencia de hepatotoxinas.

\section{MATERIALES Y METODOS}

Lugares de estudio: Los tres cuerpos de agua dulce seleccionados para el muestreo corresponden a lugares con importancia para la comunidad debido a que son utilizados para diferentes propósitos: Lago Peñuelas, ubicado en Valparaíso y Tranque Los Aromos, ubicado en Limache, son empleados como fuente agua tanto para abastecimiento público como recreacional; Tranque Recreo, ubicado en Villa Alemana, presenta sólo uso recreacional. Cada cuerpo de agua fue muestreado mensualmente durante un período de 11 meses, siendo el $1^{\circ}$ muestreo realizado en el mes de Marzo 2005 y el último en el mes de Enero del 2006.

Recolección de muestras: En cada cuerpo de agua se realizó un muestreo de superficie con una red planctónica con tamaño de poro de 55ìm (Hydro-Bios, Kiel-Holtenau). Cada muestra de agua fue concentrada en botellas de borosilicato estériles de $250 \mathrm{~mL}$ y transportadas en cajas refrigeradas con hielo a temperatura controlada.

Determinación de parámetros abióticos: En cada cuerpo de agua se realizó la medición de temperatura y conductividad utilizando un coductímetro YSI modelo 33 portátil.

Identificación taxonómica: Cada muestra fue analizada utilizando un microscopio de luz (Zeiss Standard 25) a través de un montaje húmedo. La identificación a nivel de género fue realizada de acuerdo a Bergey's Manual of Systematic Bacteriology, edición 2001.

Detección de cianotoxinas: Las muestras fueron filtradas con la misma malla de muestreo con el propósito de extraer el agua. Los concentrados fueron puestos en tubos eppendorf y refrigerados a $-20^{\circ} \mathrm{C}$, para su posterior liofilización con un liofilizador Lyovac GT2. Las muestras liofilizadas fueron analizadas por espectrometría de masas MALDI-TOF MS.

\section{RESULTADOS}

La temperatura y conductividad presentaron una variación estacional, registrándose los valores más altos en los períodos de primavera-verano, sin embargo, estas variaciones no presentaron mayor importancia para la formación de florecimientos, ya que éstos fueron encontrados durante todo el período de muestreo.

Morfológicamente se identificaron géneros de cianobacterias en todos los cuerpos de agua seleccionados. En las muestras de Lago Peñuelas se identificaron tres géneros productores de toxinas: Anabaena, Microcystis (Fig. 1-e) y Oscillatoria, y un género no productor de toxinas el cual correspondió a Chroococcus. En las muestras del Tranque Los Aromos se identificaron cuatro 
géneros productores de toxinas: Anabaena (Fig 1-e), Anabaenopsis, Microcystis y Oscillatoria, y un género no productor de toxinas: Spirulina. Por último en las muestras del Tranque Recreo, se identificaron tres géneros productores de toxina los cuales correspondieron a Anabaena, Microcystis y Oscillatoria (Fig 1-f) y un género no productor de toxinas (Spirulina).

También se detectaron florecimientos de cianobacterias en los tres cuerpos de agua. En el Lago Peñuelas (Fig.1-a) se detectó florecimientos de Microcystis en Diciembre de 2005 y Enero de 2006. En Embalse Los Aromos (Fig. 1-b) se detectó un florecimiento de Microcystis en el mes de Junio de 2005. Finalmente en el Tranque Recreo (Fig. 1-c) se detectaron florecimientos de Oscillatoria, en Marzo, Mayo, Agosto, Septiembre y Noviembre del año 2005; y de Anabaena en Enero del 2006.

Por último en los meses de Octubre y Diciembre del año 2005 se detectaron dos florecimientos dominados por ambos géneros, Anabaena y Oscillatoria (Tabla 1).

Por espectrometría de masas MALDI-TOF, se determinó cualitativamente la presencia de: microcistina -LA, -LR, -RR, -YR y nodularina (Tabla 2), además de la presencia de péptidos no tóxicos (Tabla 3).

\section{DISCUSION}

En el presente trabajo se pudo confirmar que los cuerpos de agua dulce de la $\mathrm{V}$ Región en estudio contienen géneros de cianobacterias que pueden producir péptidos tóxicos y/o no tóxicos, y además se detectó en todos ellos la presencia de hepatotoxinas. En Tranque Recreo y Lago Peñuelas se detectó la presencia de microcistina-LA en una muestra de Mayo del año 2005 y la presencia de microcistina-RR y -LA en Julio del año 2005 respectivamente (Tabla 2). En ambos casos el género productor de cianotoxinas que se observó en la muestra fue Oscillatoria, sin embargo, a diferencia de la muestra del Tranque Recreo, a la cual se le puede adjudicar la producción de la toxina únicamente a este género debido a la presencia de un florecimiento, la muestra del Lago Peñuelas, en esta fecha, presentaba una gran diversidad de microalgas con bajas densidades, por lo que la existencia de otro género productor de toxinas podría estar presente.

Vera (2005), realizó un estudio en cuerpos de agua de la $\mathrm{V}$ región donde identificó la presencia de los géneros Microcystis y Anabaena conjuntamente con microcistina-RR en una muestra del Lago Peñuelas, sin embargo, no detectó cianotoxinas en muestras que contenían Oscillatoria, como ocurrió en este estudio. Esto puede deberse en primer lugar, a que en un mismo género puede encontrarse cepas tóxicas como no tóxicas (Vezie et al., 1998) y que la presencia de una u otra puede variar en el tiempo dependiendo de las condiciones de cada cuerpo de agua (Vezie et al., 2002); y en segundo lugar puede que simplemente la densidad de Oscillatoria haya sido mayor durante el período de estudio de este trabajo, lo que permitió detectar las cianotoxinas con mayor facilidad.

Tabla 1: Géneros de cianobacterias encontrados durante el período de muestreo. X: presencia /f: florecimiento

\begin{tabular}{|c|c|c|c|c|c|c|c|c|c|c|c|}
\hline \multirow{2}{*}{ Géneros } & \multicolumn{11}{|c|}{ Meses } \\
\hline & Mar & Ab & May & Jun & Jul & Ag & $\mathbf{S}$ & $\mathbf{O}$ & $\mathbf{N}$ & $\mathbf{D}$ & $\mathbf{E}$ \\
\hline \multicolumn{12}{|l|}{ Lago Peñuelas } \\
\hline Anabaena & $\mathrm{x}$ & & & & & & & & & & \\
\hline Chroococcus & & & & & $\mathrm{x}$ & & & & & & \\
\hline Microcystis & $\mathrm{x}$ & & & & & & $\mathrm{x}$ & & & $\mathrm{xf}$ & $\mathrm{xf}$ \\
\hline Oscillatoria & & & & & $\mathrm{x}$ & $\mathrm{x}$ & & & & & \\
\hline \multicolumn{12}{|c|}{ Embalse Los Aromos } \\
\hline Anabaena & $\mathrm{x}$ & $\mathrm{x}$ & & & & & & & & & \\
\hline Anabaenopsis & $\mathrm{x}$ & $\mathrm{x}$ & & & & & & & & & \\
\hline Microcystis & $\mathrm{x}$ & $\mathrm{x}$ & & $\mathrm{xf}$ & & & $\mathrm{x}$ & $\mathrm{x}$ & & $\mathrm{x}$ & $\mathrm{x}$ \\
\hline Oscillatoria & $\mathrm{X}$ & $\mathrm{X}$ & & $\mathrm{x}$ & & & $\mathrm{x}$ & & & $\mathrm{X}$ & $\mathrm{x}$ \\
\hline Spirulina & $\mathrm{x}$ & & & & & & & & & & \\
\hline \multicolumn{12}{|l|}{ Tranque Recreo } \\
\hline Anabaena & $\mathrm{x}$ & & & & & & $\mathrm{x}$ & $x \mathrm{f}$ & & $x \mathrm{ft}$ & $x f$ \\
\hline Microcystis & $\mathrm{x}$ & & & & & & & & & & \\
\hline Oscillatoria & $\mathrm{xf}$ & & $\mathrm{xf}$ & & & $\mathrm{xf}$ & $\mathrm{xf}$ & $\mathrm{xf}$ & $\mathrm{xf}$ & $\mathrm{xf}$ & $\mathrm{x}$ \\
\hline Spirulina & $\mathrm{x}$ & & & & & & & & & & $\mathrm{x}$ \\
\hline
\end{tabular}


Tabla 2: Cianotoxinas presentes en las muestras

\begin{tabular}{|l|l|l|}
\hline $\begin{array}{l}\text { Lugares de } \\
\text { muestreo }\end{array}$ & $\begin{array}{l}\text { Cianobacterias } \\
\text { presentes }\end{array}$ & \multicolumn{1}{c|}{$\begin{array}{c}\text { Cianotoxinas } \\
\text { presentes }\end{array}$} \\
\hline $\begin{array}{l}\text { Embalse } \\
\text { Los } \\
\text { Aromos }\end{array}$ & $\begin{array}{l}\text { Anabaena, } \\
\text { Anabaenopsis, } \\
\text { Microcystis, } \\
\text { Oscillatoria, } \\
\text { Spirulina }\end{array}$ & $\begin{array}{l}\text { Microcistina-LR, } \\
\text { RR, YR } \\
\text { Nodularina }\end{array}$ \\
\hline $\begin{array}{l}\text { Tranque } \\
\text { Recreo }\end{array}$ & Oscillatoria & Microcistina-LA \\
\hline $\begin{array}{l}\text { Lago } \\
\text { Peñuelas }\end{array}$ & $\begin{array}{l}\text { Chroococcus, } \\
\text { Oscillatoria }\end{array}$ & Microcistina-RR, LA \\
\hline
\end{tabular}

\begin{tabular}{|l|l|l|}
\hline $\begin{array}{l}\text { Lugar de } \\
\text { muestreo }\end{array}$ & $\begin{array}{l}\text { Cianobacterias } \\
\text { presentes }\end{array}$ & \multicolumn{1}{|c|}{$\begin{array}{c}\text { Cianotoxinas } \\
\text { presentes }\end{array}$} \\
\hline $\begin{array}{l}\text { Embalse } \\
\text { Los } \\
\text { Aromos }\end{array}$ & $\begin{array}{l}\text { Anabaena, } \\
\text { Anabaenopsis, } \\
\text { Microcystis, } \\
\text { Oscillatoria, } \\
\text { Spirulina }\end{array}$ & $\begin{array}{l}\text { Microcina SF608, } \\
\text { Microginina Fr1 o } \\
\text { Fr3 }\end{array}$ \\
\hline $\begin{array}{l}\text { Tranque } \\
\text { Recreo }\end{array}$ & Oscillatoria & $\begin{array}{l}\text { Aeruginosina, } \\
\text { Cianopeptolina, } \\
\text { Anabaenopeptina B }\end{array}$ \\
\hline $\begin{array}{l}\text { Lago } \\
\text { Peñuelas }\end{array}$ & $\begin{array}{l}\text { Chroococcus, } \\
\text { Oscillatoria }\end{array}$ & $\begin{array}{l}\text { Abaenopeptina B, } \\
\text { Cianopeptolina, } \\
\text { Microcina SF608, } \\
\text { Cianopeptolina SS, } \\
\text { Aeruginosina }\end{array}$ \\
\hline
\end{tabular}

Tabla 3: Péptidos no tóxicos presentes en la muestra
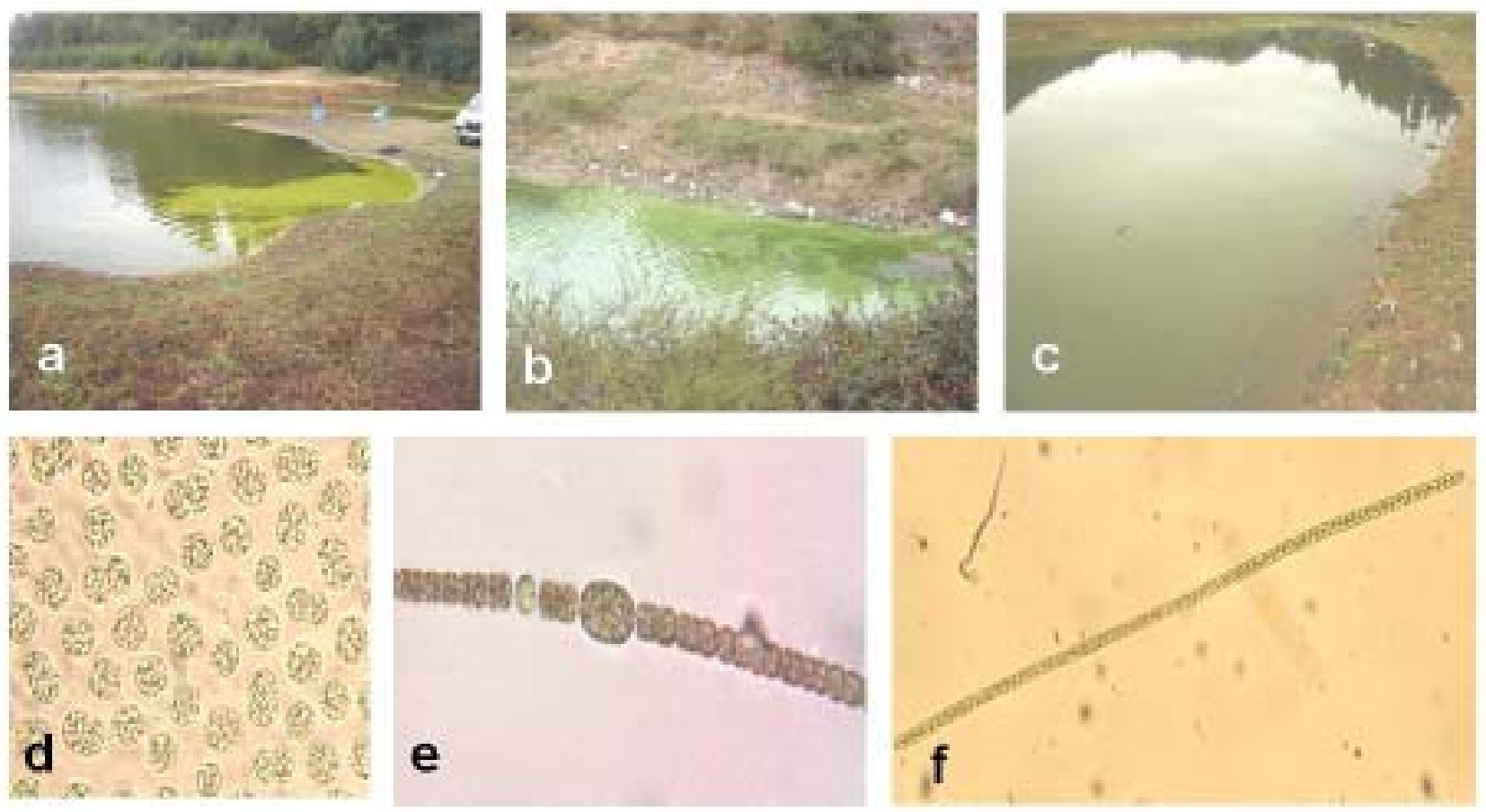

Florecimientos de Cianobacterias encontrados en los cuerpos de agua: a, Microcystis, Lago Peñuelas; b, Microcystis, Embalse Los Aromos; c, Oscillatoria, Tranque Recreo d, Microcystis sp. (2000x), Lago Peñuelas; e, Anabaena sp. (1300x), Embalse Los Aromos; f, Oscillatoria sp. (800 x), Tranque Recreo

En el Tranque Recreo se detectó la presencia de florecimientos de Oscillatoria durante cuatro meses seguidos (Tabla 1), donde la mayor concentración de filamentos no se encontraba en la superficie, sino más bien en las capas subsuperficiales. Esto coincide con estudios que indican que este género es capaz de formar florecimientos en las capas más profundas de la columna de agua, debido a su preferencia a bajas temperaturas e intensidades de luz, además de la presencia de vesículas de gas capaces de soportar altas presiones (Konopka, 1981; Mur et al., 1999; Bright \& Walsby, 1999).

En el caso de este cuerpo de agua en particular, esto es importante debido a que la mayoría de los bañistas que lo utilizan no se percatan de la alta densidad de 
cianobacterias presentes y realizan sus actividades recreativas sin ningún reparo. A pesar de que es poco probable una intoxicación aguda sólo mediante la natación, la capacidad de las hepatotoxinas de promover la aparición de tumores a largo plazo, presupone igualmente un riesgo para aquellas personas que consumen pequeñas dosis de agua involuntariamente durante el baño (Sivonen et al., 1999).

El Embalse Los Aromos fue el cuerpo de agua donde se encontró la mayor diversidad de hepatotoxinas. En Abril del 2005 se detectó microcistina-LR, -RR, -YR y nodularina, esto coincide con la gran cantidad de géneros tóxicos existentes en la muestra (Anabaena, Anabaenopsis, Microcystis y Oscillatoria), lo que impide atribuir la producción de estos péptidos a un género en particular (Tabla 2), en especial en el caso de la nodularina, ya que según la literatura el único género conocido que produce esta toxina es Nodularia; este péptido es muy estable y puede llegar a durar libre en el medio acuático por más de nueve días (Mazur \& Plinski, 2001; Metcalf \& Codd, 2004).

Los parámetros abióticos medidos durante el periodo de estudio, temperatura y conductividad, no presentaron una mayor importancia para la formación de florecimientos, debido a que: i) se detectaron florecimientos de cianobacterias tanto a temperaturas cercanas a la mínima como a la máxima registrada y, ii) la conductividad en cada cuerpo de agua no presentó variaciones significativas durante el periodo de muestreo.

El Lago Peñuelas abastece de agua para consumo a la ciudad de Valparaíso, mientras que el Embalse Los Aromos es utilizado para abastecer a la región en situaciones de emergencia por déficit de agua del río Aconcagua.

El hecho de que en ambos cuerpos de agua se haya detectado florecimientos de cianobacteria que producen péptidos tóxicos y no tóxicos, metabolitos que son químicamente muy estables en el ambiente, revela el riesgo en el cual se encuentra la población que consume el agua (Lawton \& Robertson, 1999), más aún cuando las plantas de tratamiento no presentan un protocolo específico para la eliminación de éstos péptidos en los reservorios.

En el año 1997 la Organización Mundial de la Salud estableció un valor provisional para Consumo Diario Tolerable (CDT) de 1,0 ìg/l de microcistina-LR para aguas de abastecimiento público (citado en Weckesser et al., 1999; Roset et al., 2001), por lo que en Chile, es necesario promover normativas para la calidad del agua respecto a la presencia de cianotoxinas y planes de monitoreo que permitan detectar y/o evitar la formación de florecimientos.

\section{CONCLUSIONES}

Se detectó la presencia de géneros de cianobacterias tóxicas y no tóxicas en los tres cuerpos de agua muestreados.

Se registra en este estudio la presencia de florecimientos de Microcystis sp. en Lago Peñuelas y en el Embalse Los Aromos y de Anabaena y Oscillatoria en el Tranque Recreo.

Se detectó la producción de las hepatotoxinas microcistina-LA y -RR en el Lago Peñuelas, microcistinaLA en Tranque Recreo y microcistina-LR, -RR, -YR y nodularina en Embalse Los Aromos, siendo este el primer reporte de nodularina en Chile.

Se determinó, adicionalmente la producción de péptidos no tóxicos biológicamente activos en los tres cuerpos de agua dulce muestreados.

\section{REFERENCIAS}

Bartram, J.; Carmichel, W.; Chorus, I.; Jones, G.; Skulberg, O. (1999). Introduction. In I Chorus and J. Bartram (eds), Toxic Cyanobacteria in Water: A guide to their public health consequences, monitoring and management. E and F. N. Spon, London.

Bright, D. \& Walsby, A. (1999). The relationship between critical pressure and width of gas vesicles in isolates of Planktothrix rubescens from Lake Zürich. Microbiology. 145:2769- 2775

Campos,V.; Cantarero, S.; Urrutia, H.; Heinze, R.; Wirsing, B.; Neumann, U. (1999). Microcystin in Cyanobacterial Blooms in a Chilean Lake. System. Appl. Microbiol. 22:169-173

Campos, V.; Lisperguer, S.; Weckesser, J.; Vera, A.; Muñoz, D. (2005). Cianobacterias y Riesgos Potenciales de Toxicidad en Aguas Continentales de Chile. Boletín Micológico. Vol 20:73-81

Carmichel, W. (1994). The toxins of cyanobacteria. Scientific American. 270:78-86

Carmichel, W.; Azevedo, S.; An, j.; Molica, R.; Jochimsen, E.; Lau, S.; Reinhart, G.; Eaglesham, G. (2001). Human Fatalities from Cyanobacteria: Chemical and Biological Evidence for Cyanotoxins. Enviromental Health Perspectives. 109:663-667

Castenholz, R. (2001). Phylum BX. Cyanobacteria. 473-487 pp. In N.R. Krieg and S.G. Holt (eds), Bergey's Manual of Systematic Bacteriology. Vol I. The Williams and Wilikins Co. Baltimore.

Jochimsen, E.; Carmichel, W.; An, J.; Cardo, D.; Cookson, S.; Holmes, M.; de Melo Filho, D.; Lyra, T.; Spinelli, V.; Azevedo, S.; Jarvis, W. (2004). Liver Faliure and Death After Exposure to Microcystins at a Hemodialysis Center in Brazil. New England Med. 338:873-878

Konopka, A. (1981). Influence of Temperature, Oxygen and pH on a Metalimnic Population of Oscillatoria rubescens. App. and Environ. Microbiology 42:102-108

Lawton, L. \& Robertson, K. (1999). Physico-chemical treatment 
methods for the removal of microcystins (cyanobacterial hepatotoxins) from potable waters. School of Applied Sciences. The Robert Gordon University. Chem. Soc. Rev. 28:217-224

Mazur, H. \& Plinski, M. (2001). Stability of cyanotoxins, Microcystin-LR, Microcystin- RR and Nodularin in Seawater and BG-11 Medium of Different Salinity. Oceanologia 43:329-333

Metcalf, J. \& Codd, G. (2004). Cyanobacterial Toxins in The Water Enviroment. Review of Current Knowledge FR/R009. Foundation for Water Research, Allen House, The Listons, 36pp.

Mur, L.; Skulberg, M. \& Utkilen, H. (1999). Cyanobacteria in the Enviroment. In I Chorus and J. Bartram (eds), Toxic Cianobacteria in Water: A guide to their public health consequences, monitoring and management. E and F. N. Spon, London.

Neuman, U.; Campos, V.; Cantarero, S.; Urrutia, H.; Henzie, R.; Weckesser, J.; Erhard, M. (2000). Co-ocurrence of Non-toxic (Cyanopeptolin) and Toxic (Microcistin) Peptides in a Bloom of Microcystis sp. From a Chilean Lake. Syst. And App. Microbiology 23:191-197

Paerl, H. W. (1996). A Comparison of Cyanobacterial Bloom dynamics in freshwater, estuarine and marine environment. Phycologia. 35:2535.

Rippka, R.; Watebury, J. \& Herdman, M. (2001). Subsection IV (Nostocales). 562-565 pp. In N.R. Krieg and S.G. Holt (eds), Bergey's Manual of Systematic Bacteriology. Vol I. The Williams and Wilikins Co. Baltimore.
Roset, J.; Aguayo, S. \& Muñoz, M. (2001). Detección de Cianobacterias y sus Toxinas. Una Revisión. Toxicol. 18:65-71

Sivonen, K \& Jones G. (1999). Cyanobacterial toxins. In I Chorus and J. Bartram (eds), Toxic Cianobacteria in Water: A guide to their public health consequences, monitoring and management. $\mathrm{E}$ and $\mathrm{F}$. N. Spon, London.

Ueno,Y.; Nagata, S.; Tsutsumi,T.; Hasegawa, A.; Watanabe,MF.; Park, H-D.; Chen, G-C.; Chen, G.; Yu, S-Z. (1996). Detection of Microcystins, a blue-green algal hepatotoxin, in drinking water sampled in Haimen and Fusui, endemic areas of primary liver cancer in China, by highly sensitive immunoassay. Carcinogenesis 17:1317-1321

Vera, A. (2005). Determinación de Cianobacterias y Cianotoxinas en Cuerpos de Agua Dulce de la V Región, Chile. Tesis para optar al Título de Biólogo. Pontificia Universidad Católica de Valparaíso.

Vézie, C. ; Brient, I. ; Sivonen, K. ; Bertu, G. ; Lefeuvre, J. ; Salkinoja, M. (1998). Variation of Microcistin Content of Cyanobacterial Blooms and Isolated Strains in Lake Grand- Lieu (France). Microbial Ecology. 35:126-135

Vézie, C.; Rapala, J.; Vaitomaa, J.; Seitsonen, J.; Sivonen, K. (2002). Effect of Nitrogen and Phosphorus on Growth of Toxic and Non Toxic Microcystis Strains and on Intracellular Microcistin Concentration. Microbial Ecology. 43:443-454

Weckesser, J.; Campos, V.; Ramos, A.; Neumann, U. (1999). Biologically Active Peptides in Cyanobacteria. Boletín Micológico. 14:19-29 